\title{
Bias Magnetic Field of Stack Giant Magnetostrictive Actuator: Design, Analysis, and Optimization
}

\author{
Zhaoshu Yang, Zhongbo He, Dongwei Li, and Ce Rong \\ Department of Vehicle and Electrical Engineering, Shijiazhuang Mechanical Engineering College, Shijiazhuang 050003, China \\ Correspondence should be addressed to Zhaoshu Yang; yangzhaoshu@sina.cn
}

Received 29 March 2016; Revised 15 June 2016; Accepted 16 June 2016

Academic Editor: Giovanni Berselli

Copyright (C) 2016 Zhaoshu Yang et al. This is an open access article distributed under the Creative Commons Attribution License, which permits unrestricted use, distribution, and reproduction in any medium, provided the original work is properly cited.

\begin{abstract}
Many novel applications using giant magnetostrictive actuators (GMA) require their actuators output bidirectional strokes to be large enough to drive the load. In these cases, the sophisticated method to form such a sufficient bias field with minimum power and bulk consumption should be considered in the principal stage of GMA design. This paper concerns the methodology of bias field design for a specific GMA with stack PMs and GMMs (SGMA): both loop and field models for its bias field are established; the optimization method for given SGMA structure is outlined; a prototype is fabricated to verify the theory. Simulation and test results indicate that the bias field could be exerted more easily using SGMA structure; the modeling and optimization methodology for SGMA is valid in practical design.
\end{abstract}

\section{Introduction}

In the development of novel hydraulic servo valve (EHSV) serving in aerospace industry, giant magnetostrictive actuator (GMA) is a thriving device to generate micro stage displacement fast and precisely [1-5]. In many cases, a static bias field is employed in GMA to give a preelongation of the magnetostrictive rod when a bidirection stroke is required. Since an appropriate bias field helps these devices to operate in a region, both slope and linearity of magnetostriction are favorable and avoid the specific "double-frequency" characteristic of GMM under alternating drive $[6,7]$. There are mainly two ways to provide such a bias field for GMM devices: (a) by adding a secondary DC coil or a DC offset to the AC current and (b) by using permanent magnets (PM).

Each method has its own advantages and disadvantages. Method (a) is easier to be realized and the field intensity is more readily to be adjusted using this method, but it consumes more power and therefore increases the total power losses. Furthermore, two coils within in GMA will induce mutual inductance reducing the driving efficiency and enlarge the total radius dimension considerably. For instance, Joshi and Kadoli developed a DC biased GMA with two concentric coils; inductance for both coils with GMM core is, respectively, $16.45 \mathrm{mH}$ and $18.3 \mathrm{mH}$, the flux leakage of outer coil reaches $12 \%$, and overall radius of outer coil is 4.1 times that of GMM rod [8]. On the other hand, providing bias field through PM requires no electrical power and therefore is more preferred in recent developments $[9,10]$. However, unlike the DC bias method, bias field from PM cannot be easily tailored, weaker magnets tend to fail their purpose while stronger ones drive GMM to saturation, and it is always challenging to find magnets of appropriate remanent flux density, size, and shape. In most of the cases, the configuration of $\mathrm{PMs}$ is designed and costume-made at preliminary stage to match the actuator.

The traditional configuration for PM bias in GMA is based on "tube-like" magnets. With a GMM rod located in the innermost of the PM tube, this configuration helps to form a uniformed longitudinal field all through GMM bar [11]. However, when longer and thicker GMM rods are used, significant phenomena such as saturation and end effects are likely to occur [12]; the magnetic bias may be subject to conditions outside of the transducer. That is, external ferromagnetic materials in close proximity to the transducer can divert magnetic flux away from GMM rod, reducing magnetic bias [13]. In order to offer a more uniform bias field to long GMM bars, Zhang et al. have developed a novel tube-like 
PM combining a series of shorter PM rings gradually changing their shapes and remanent flux density, but this design requires an elaborated preparation for PM rings; if the GMM in the actuator changes its size, the PM rings have to be redesigned accordingly [11]. In addition, a concentric configuration of GMM bar and tube-like PM is not compatible with the common mechanical amplifier. In these designs, GMM bars are mounted orthogonally into a mechanical frame with flexible hinges $[14,15]$; the tube-like PM is usually too bulky to embed into this frame, making designers have to choose between amplifier and PM tube; Karunanidhi and Singaperumal developed two flapper-nozzle servo valves; the first one employs mechanical amplifier and the second one uses tube-like PM, and the bidirectional stroke of the mechanical amplified one could only be realized using DC bias [3]; moreover, for servo valves demanding high reliability, they tend to employ redundant structures in their electromechanical transducers [16]; when the redundant actuators are parallelly located, their radius size should be limited and there will be little space for tube-like PMs.

Another configuration employed distributed PMs to provide bias field; PM patches are usually located on poles of GMM bar. This configuration helps to relieve the end effects of long GMM rod; the disk shape PMs are placed in line with GMM rod which saves a lot of transversal dimension and therefore being more easily to be located within a mechanical amplifier. Braghin et al. developed a low frequency magnetostrictive inertial actuator for vibration control with this configuration, which reduces the eigenfrequency of actuator and extends its stroke; this configuration also helps to reduce the general size of actuator thus allowing to mount it even in narrow cavities [17]; in addition, compared with tube-like PM, this configuration provides sufficient bias field with limited quantity of PM [18], reducing the total mass of actuator. This characteristic is significant for devices working in aerospace industry [19].

Despite the potential of distributed PMs in GMA design, the most crucial problem of this arrangement is that the field intensity will not be uniformed along the rod, especially when the aspect ratio for GMM is large. How to gain an acceptable homogeneous bias field in long GMM rod with little bulk penalty has become a hot point for recent researchers. Zhang et al. developed a triple-ring PM structure, in their design; three PM rings are located surrounding both ends and the center of GMM rod; in this way the field homogeneity was greatly improved for GMM rod with an aspect ratio larger than 3.5 [11]; Mu et al. simulated three different PM arrangements in GMA design and provide some qualitative conclusion on distributed PM selection [20]; Xia et al. compared two typical distributed PM structures, the in-line structure and coaxial structure, using FEM; their research work indicates that, for equal aspect ratio, the in-line configuration will provide larger field intensity while the coaxial arrangement is more uniformed in field distribution [21]; Choi et al. proposed simultaneous configuration optimization methods for PMs in magnetostrictive sensors; they combine FEM and topological analysis to determine the optimized shape for PMs and yokes $[22,23]$.
Our institute focuses on the development of novel electrohydraulic servo valves served in aeronautic applications, which require the actuator to output a large bidirectional stroke precisely. According to the above analysis, the bias field implementation for this specific case is a little troublesome for traditional PM layout: a tube-like magnet will make it bulky and magnet pairs on both ends cannot ensure a uniformed field distribution. In this paper, we employ a configuration which geometrically iterates the poles PM case; that is, pole magnets are located in series and alternatively with short GMM patches; in this way the aspect ratio for each GMM patch is not so large and the transversal dimension could be saved; this structure is named as stack GMA (SGMA) because of its specific structure [13]. However, although this structure has been proposed for several years and exhibits some exciting merits as mentioned, there are limited works concerning its general design and analysis. For comparison, Figure 1 provides 3D models of pole GMA and SGMA structure.

Generally, there are mainly two different analysis methods for magnetic field in ferromagnetic materials, namely, loop method and field method. Loop analysis is conducted based on the assumption that field distribution is homogeneous in radius span; therefore, the GMM rod could be modeled in its longitudinal direction as a network of magnetic circuits [24]; then Kirchhoff's Laws are employed to calculate the field intensity and flux density; in traditional analysis, the magnetic circuit of GMA is regarded as a single magnetic loop $[1,19]$, since in a classical tube-like PM configuration, the bias field is assumed to be uniformly distributed. Loop analysis denotes a lumped element method (LEM), in which the field calculated distribution is rough and noncontinuous, while this method is concise in structure, easily deducing an analytical solution, since only algebraic equations are resolved. Consider that the constitutive model for GMM devices is usually fully multiphysically coupled; this approach could reduce its overall complicity, which is more readily to be embedded into a practical controller as a feed-forward inverse [2, 25, 26]; this method also provide an explicit parametrical expression of magnetic field, which assists subsequent parametrical optimization and identification $[27,28]$.

Field method denotes a more precise analysis using FEM package. The analysis is based on Maxwell equation; the field distribution is calculated by resolving partial differential equations simultaneously on each node. It provides a continuous description on field distribution but consumes more computing resource. This method also fails to get an overall parametrical solution, let alone parametrical optimization and identification. Therefore, this method is more attractive if we are only interested in field analysis of a certain structure $[10,29]$.

In this paper, we focus on the specific layout of SGMA; both loop and field models for its particular bias field are built. Considering the unevenness of axial direction, more magnetic circuits are employed. A general model for bias field in SGMA is built. This model is compatible with arbitrary GMM number and arbitrary number of divided loops in each GMM bar. Therefore, it provides guidance for SGMA design. In field analysis, a more precise field distribution is studied using FEM for different design parameters. The study results of field 


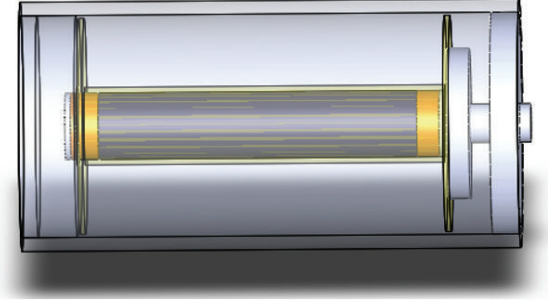

(a) Pole GMA

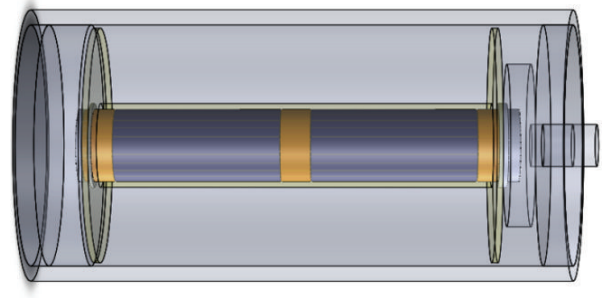

(b) Stack GMA

Figure 1: 3D model of pole GMA and stack GMA.

analysis are proved to be supportive and compensable for loop analysis. A specific superposition effect of multiple PMs is observed. Dealing with this superposition effect, an optimization for SGMA structure is conducted. This paper provides basic analysis for SGMA which could be used as a premier guide for its design; the loop model can be coupled easily with magnetization and magnetostriction procedures in order to study its overall dynamics.

The structure of this work is outlined as follows: Section 1 covers the introduction of the specific structure of SGMA and its corresponding bias field analysis method; Section 2 concerns the design and analysis of the single GMM case, since this case represents the basic structure of SGMA; loop and field analysis are employed in this section; their results are compared and discussed; Section 3 extends the basic analysis in single GMM case into multi-GMM case; in loop analysis the general equation for SGMA bias field design is compatible with any GMM quantity and loop number; in field analysis, the superposition effects are observed; in order to improve the field homogeneity, an optimization design was conducted; in Section 4, a series of magnetic and displacement tests are conducted to validate the design and optimization result. In Section 5, some conclusions are achieved concerning the roadmap of SGMA design and its specific characteristics.

\section{Single GMM Structure}

The single GMM structure includes a long GMM bar with PM patches attached on its both ends. This structure stands for the basic element of a SGMA, since the multi-GMM case could be regarded as a structure iteration of this basic structure. Unlike the traditional configuration, GMM bar is located within the center of a tube-like PM or DC current coil; in SGMA, GMM bar is embedded between a pair of PM patches [30, 31]; the bias field distribution alters considerably along the axial position.

\subsection{Loop Analysis}

2.1.1. Setting Up Loop Model. The loop analysis is based on the magnetic circuit theory. As it is stated above the unevenness of bias field makes it necessary to divide the rod into several segments. In most of the previous works [11, 18], the GMM rod is divided into three loops. The first loop combines a PM and its adjacent GMM part, another loop is formed by the middle GMM part, and the last loop is formed by the PM and

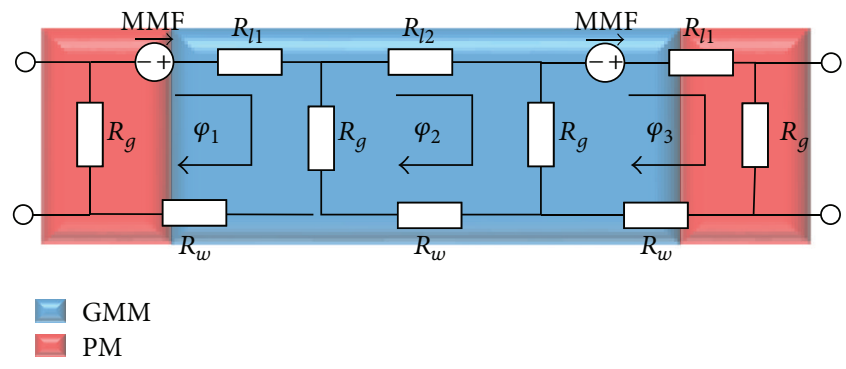

FIgURE 2: 3D diagram for an element and its equivalent magnetic circuit.

its adjacent GMM part on the other end. The GMM parts in above three loops are all of equal size. The overall circuit model is exhibited as Figure 2.

In Figure 2, $R_{l 1,2}$ denotes the magnetic reluctance of the rod within each loop formed by GMM and PM patches alternatively; $R_{g}$ and $R_{w}$ denote, respectively, the magnetic reluctance of the air gap from GMM bar to wall and the reluctance of wall; MMF denotes magnetomotive force provided by PM; based on Ampere's Law, the above parameters could be expressed as

$$
\begin{aligned}
\mathrm{MMF} & =H_{c} l_{\mathrm{PM}}, \\
R_{l 1} & =R_{\mathrm{PM}}+\frac{1}{3} R_{\mathrm{GMM}}, \\
R_{l 2} & =\frac{1}{3} R_{\mathrm{GMM}} .
\end{aligned}
$$

The expression of magnetic reluctance in each section is defined as

$$
R=\int \frac{d l}{\mu_{0} \mu_{r} A_{\mathrm{GMM}}} .
$$

In the above equation, $\mu_{r}$ denotes the relative magnetic permeability of a certain component, which is determined as the experimental data as Figure 3 , and $A_{\mathrm{GMM}}$ denotes the corresponding section area; in GMM, the nonlinearity of its magnetic reluctance is counted by employing a $B(H)$ curve achieved from practical tests.

In previous works [18], the governing equations for each magnetic circuit are set up based on nodal current analysis; there are 6 formulas in the governing equation to determine 


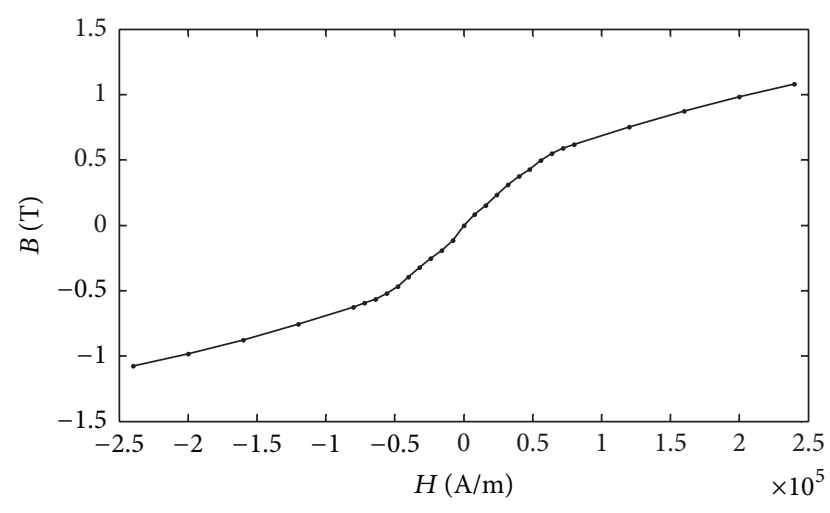

FIgURE 3: Practical test curves of GMM magnetization.

magnetic flux. In this work, the governing equation is established using mesh current analysis; compared with the nodal current method, only three independent equations are solved simultaneously in a single element; this method reduces the order of constitutive equation.

In mesh current analysis, the state variables are selected as the fluxes flowing within each mesh loop $\varphi_{1-3}$; with respect to the boundary, its flux could be calculated as the flux differential of abutting meshes. Its governing equation is therefore built as

$$
\begin{aligned}
R_{l 1} \varphi_{1}+R_{g}\left(\varphi_{1}-\varphi_{2}\right)+R_{w} \varphi_{1}+R_{g} \varphi_{1} & =\mathrm{MMF}, \\
R_{l 2} \varphi_{2}+R_{g}\left(\varphi_{2}-\varphi_{3}\right)+R_{w} \varphi_{2}+R_{g}\left(\varphi_{2}-\varphi_{1}\right) & =0, \\
R_{l 1} \varphi_{3}+R_{g} \varphi_{3}+R_{w} \varphi_{3}+R_{g}\left(\varphi_{3}-\varphi_{2}\right) & =\mathrm{MMF} .
\end{aligned}
$$

The above equation could be transformed into a matrix form:

$$
\left(\begin{array}{ccc}
c_{1} & a & 0 \\
a & c_{2} & a \\
0 & a & c_{1}
\end{array}\right)\left(\begin{array}{l}
\varphi_{1} \\
\varphi_{2} \\
\varphi_{3}
\end{array}\right)=\left(\begin{array}{c}
\mathrm{MMF} \\
0 \\
\mathrm{MMF}
\end{array}\right)
$$

in which $a=-R_{g}, c_{1}=R_{l 1}+2 R_{g}+R_{w}$, and $c_{2}=R_{l 2}+2 R_{g}+R_{w}$.

\subsubsection{Discussion for Loop Analysis}

Field Homogeneity Analysis Based on Loop Model. Consider the 3-loop model for single GMM case (4). The unevenness could be evaluated as the differential of flux in different region:

$$
\begin{aligned}
\Delta \varphi & =\varphi_{1}-\varphi_{2} \\
& =\left(\begin{array}{lll}
1 & -1 & 0
\end{array}\right)\left(\begin{array}{lll}
c_{1} & a & 0 \\
a & c_{2} & a \\
0 & a & c_{1}
\end{array}\right)^{-1}\left(\begin{array}{c}
\mathrm{MMF} \\
0 \\
\mathrm{MMF}
\end{array}\right) .
\end{aligned}
$$

Submitted with the corresponding parameters, an explicit expression of $\Delta \varphi$ depending on $R_{\mathrm{GMM}}$ is deduced as

$$
\Delta \varphi=\frac{\operatorname{MMF}\left(R_{\mathrm{GMM}} / 3+R_{w}\right)}{R_{\mathrm{GMM}}^{2} / 9+\widehat{a}_{1} R_{\mathrm{GMM}} / 3+\widehat{a}_{0}},
$$

in which $\widehat{a}_{0}=\left(2 R_{g}+R_{w}\right) R_{\mathrm{PM}}+2 R_{g}^{2}+R_{w}^{2}$ and $\widehat{a}_{1}=R_{\mathrm{PM}}+$ $4 R_{g}+2 R_{w}$.

Some qualitative conclusion could be achieved from (6): to begin with, a larger MMF, will increase the unevenness; in addition, based on (6), the differential expression of $d \Delta \varphi / d R_{\mathrm{GMM}}$ could be easily achieved; then a conclusion could be approached as follows: if $R_{w} \geq 2 R_{g}$, the longitudinal flux unevenness $\Delta \varphi$ will follow the increase of $R_{\mathrm{GMM}}$. This statement, drawn from the loop analysis, agrees with the field analysis results which will be discussed in the following sections.

Although a 3-loop model could describe the alteration pattern of bias field within GMM bar, this description is rather rough. In order to provide a more explicit loop analysis, this paper extends this method to build a multiloop model for bias field alteration.

Consider the mesh flux equation (4); if there are more loops employed, it could be extended as $N$-order case:

$$
\left(\begin{array}{cccc}
\tilde{c}_{1} & a & 0 & 0 \\
a & \widetilde{c}_{2} & a & 0 \\
0 & a & \ddots & a \\
0 & 0 & a & \tilde{c}_{1}
\end{array}\right)_{N \times N}\left(\begin{array}{c}
\varphi_{1} \\
\varphi_{2} \\
\vdots \\
\varphi_{N}
\end{array}\right)=\left(\begin{array}{c}
\mathrm{MMF} \\
0 \\
\vdots \\
\mathrm{MMF}
\end{array}\right)
$$

in which $\widetilde{c}_{1}=(1 / N) R_{\mathrm{GMM}}+R_{\mathrm{PM}}+2 R_{g}+R_{w}$ and $\widetilde{c}_{2}=$ $(1 / N) R_{\mathrm{GMM}}+2 R_{g}+R_{w}$.

This extended model provides a more explicit description of the variation pattern of field distribution along axis position of GMM bar, especially when the aspect ratio is larger.

\subsection{Field Analysis}

2.2.1. Setting Up Field Model. The governing equation of static field within GMM bar could be expressed as

$$
\begin{aligned}
\mathbf{H} & =-\nabla \phi, \\
\nabla \cdot \mathbf{B} & =0, \\
B & =f(H) .
\end{aligned}
$$

In the above equation, $H=f(B)$ denotes the interpolation function of test $B-H$ curve and $\phi$ denotes the magnetic scalar potential. In a $2 \mathrm{D}$ axisymmetric case, (8) could be derived as the following wave equation:

$$
\frac{\partial}{\partial r}\left[\frac{1}{r} \frac{\partial(r \mathbf{A})}{\partial r}\right]-\frac{\partial^{2} \mathbf{A}}{\partial z^{2}}=0 .
$$

In the above equation, $\mathbf{A}$ denotes the magnetic vector potential.

2.2.2. Discussion for Field Analysis. The field distribution, represented by both color table and contour lines, in single GMM case is detailed in Figure 4.

According to Figure 4, the contour lines are distributed denser abutting PMs, which indicates a much sharper field variation within the abutting region. In practical design, it is 


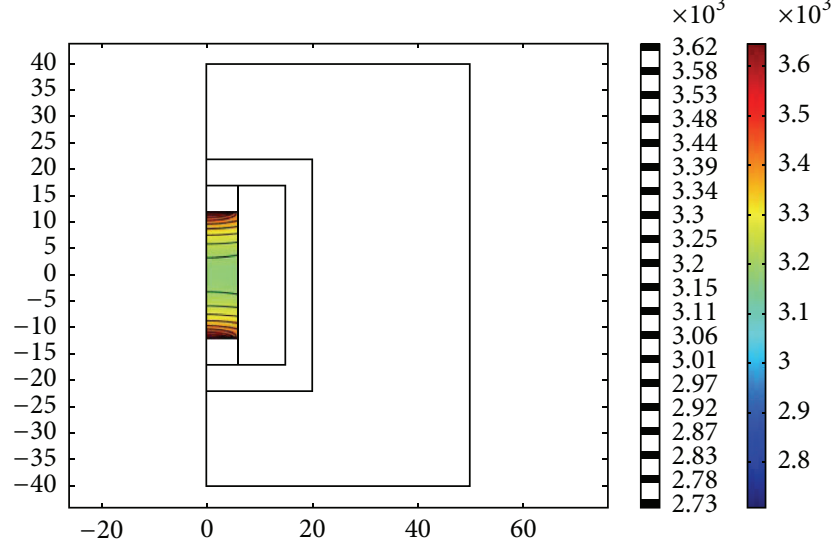

FIgURE 4: Contour plot of magnetic field distribution in single GMM case.
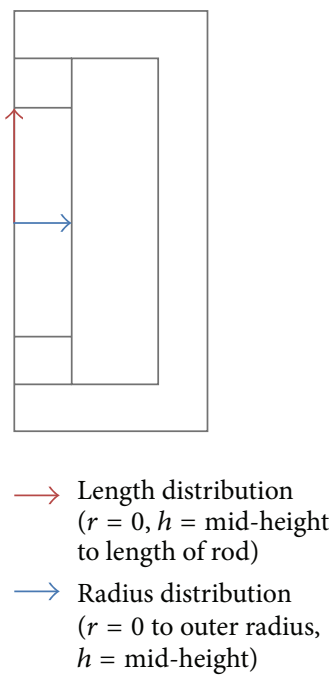

FIgURE 5: Semimajor and semiminor axis.

significant to improve the field distribution homogeneity of GMM bar. Motivated by the contour plot in Figure 4, the following factors are studied to enhance its homogeneity; the first factor is the aspect ratio of GMM geometry and the alternative factor denotes the remanent flux density of PM patches. The lengthwise and radius analysis will be conducted on the semimajor and semiminor axis, respectively, of GMM bar; their positions and directions are exhibited in Figure 5 as the arrow lines.

The comparison of magnetic field distribution, modeled by FEM as well as different loop analysis, is exhibited in Figure 6.

According to Figure 6, the axial field distribution in loop analysis gets closer to FEM result when there are more magnetic circuits employed. However, when more loops are counted, the size of the state matrix in (7) will increase, which means larger memory consumption in numerical computing, when this method is extended to multi-GMM case. In addition, the field strength will rise a little in the loops abutting the PMs; this is induced by the reducing length of each GMM

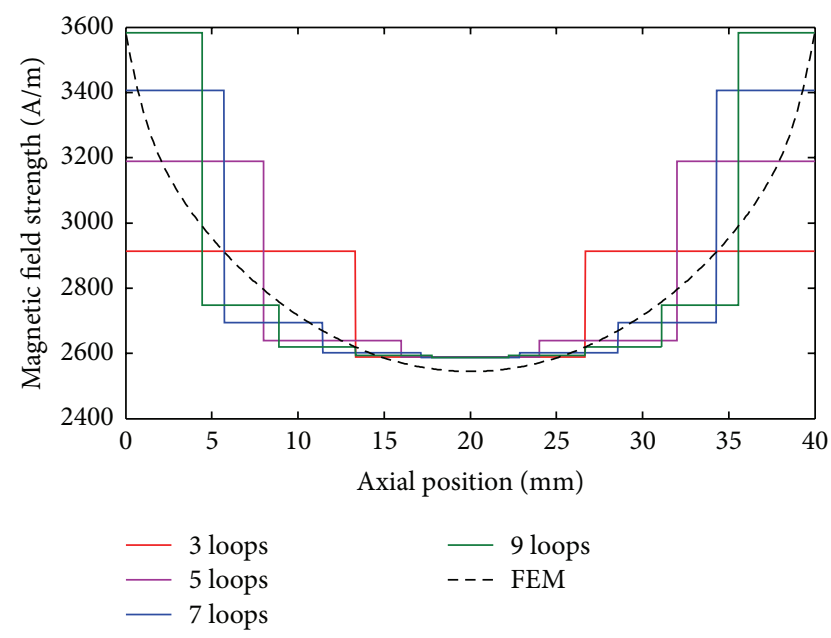

FIGURE 6: Axial field distribution described by multiloop model and FEM analysis.

patch, and a shorter length will decrease their reluctance. This phenomenon indicates that when there are more loops employed, the calculated average field will increase approaching more the real value. In addition, it should be noted that although a multiple loop analysis will approach the field analysis results, they denote two different methods in bias field analysis: in loop analysis the constitutive equation is Kirchhoff's Law, while in the field analysis, the constitutive equation is Maxwell's equation. Furthermore, the analysis is conducted for two different design parameters, namely, the aspect ratio of GMMs and remanent flux density of PMs. The analysis results are exhibited as Table 1.

The Effect of Aspect Ratio. Indicated in Table 1, as the aspect ratio increased, the field unevenness along longitudinal direction increased; on the contrary, the evenness through different locations along radius span is improved. This result provides valuable insight to practical design in SGMA and in accordance with the loop analysis result. In addition, when the aspect ratio increases, the unevenness along axial position will also increase. This result is in accordance with the loop analysis in Section 2.1.2: Since when radii are fixed, a larger aspect ratio will increase the length of GMM bar and reluctance of GMM will increase according to (2), this rise in reluctance will increase the field unevenness according to (6).

The Effect of Remanent Flux Density. According to Table 1, both the axial and radius unevenness increase when larger remanent flux density is exerted. This is also in accordance with the loop analysis: a larger remanent flux density increases MMF and eventually raises the unevenness according to (6).

Table 1 indicates that the radius evenness of all these cases is quite acceptable; this result verifies the basic assumption of loop analysis. With respect to axial evenness, between the two factors, changing of remanent flux density will greatly enhance the field evenness. But too large flux density in PM will make the ferromagnetic pole pieces subject to magnetic 
TABLE 1: Percentage difference of magnetic field along axial and radius position of GMM with different design parameters.

\begin{tabular}{lcr}
\hline Aspect ratio & Difference in axial magnetic field & Difference in radius magnetic field \\
\hline 1 & $5.03 \%$ & $0.27 \%$ \\
2 & $11.91 \%$ & $0.17 \%$ \\
4 & $28.91 \%$ & $0.12 \%$ \\
\hline Remanent flux density & Difference in axial magnetic field & Difference in radius magnetic field \\
\hline $1.2 \mathrm{~T}$ & $15.95 \%$ & $0.08 \%$ \\
$1.4 \mathrm{~T}$ & $28.91 \%$ & $0.12 \%$ \\
$1.6 \mathrm{~T}$ & $37.64 \%$ & $0.18 \%$ \\
\hline
\end{tabular}

saturation and interfere with the overall electromagnetic compatibility of GMM devices [13]; moreover, specimen of PM with larger remanent flux density is not so easy to approach in commercial market; the aspect ratio of GMM relates to the field evenness, but this factor is determined by the total strain and block force requirement of GMM devices. The above analysis also makes some indication for multi-GMM structure design and optimization which will be elaborated in Section 3.2.

\section{Multi-GMM Structure}

When the axial dimension is much larger, multi-GMM structure will be employed in SGMA design, since this structure helps to enhance the field homogeneity along GMM bar. According to the analysis in former sections, multi-GMM structure could be regarded as a structural iteration of the basic single GMM structure. Therefore, the analysis method in single GMM case could be extended to multi-GMM structure.

\subsection{Loop and Field Analysis}

3.1.1. Loop Analysis. A multi-GMM case of SGMA structure could be regarded as a structural iteration of single GMM case; a diagram for its overall magnetic circuit is simplified in Figure 7.

Based on the mesh circuit method detailed in Section 2.1.1, the governing equation for a SGMA magnetic circuit with multiple layers is expressed in a tridiagonal matrix form when $N$-loop model is employed in each single GMM layer:

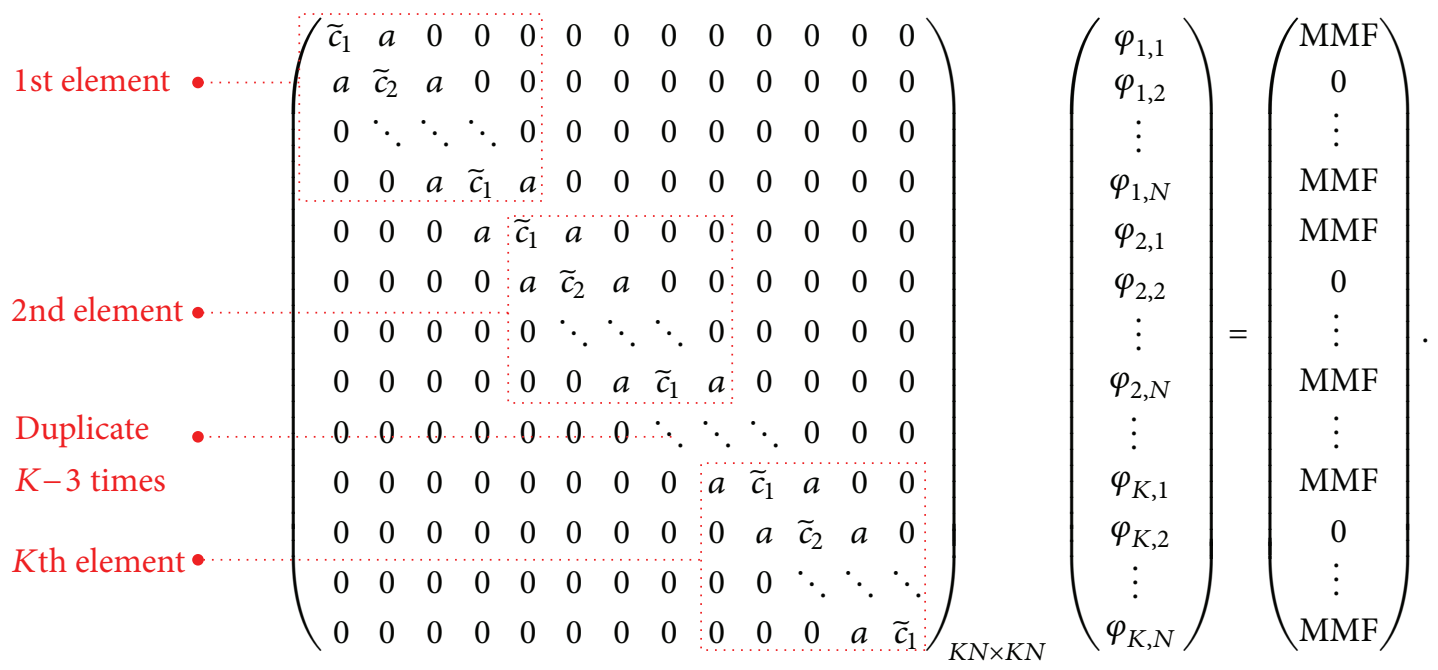

In the above equation, $\widetilde{c}_{1}$ and $\widetilde{c}_{2}$ are defined after (7); the sparse matrix indicates a simplified method to set up the governing equation of the general magnetic circuit for lineararrayed GMA structure, especially when the element quantity is too large to set up equation in every single mesh. In the constitutive matrix of (10), the first and last four rows indicate the circuit equations of first and last magnetic element; correspondingly, the 5 th to 8 th rows in (10) represent the second magnetic element; this part could be duplicated $K-3$ times when the total element number is $K$. With the help of (10), one can transform the design and manufacture of a SGMA into the computation of its governing matrix without setting up its specific loop model; an engineering issue could be represented and extended more readily in its mathematical form.

Figure 8 exhibits the loop analysis result for multi-GMM structure; for different GMM numbers and different loop model, the average magnetic field along axial position of GMM array is calculated, respectively. 


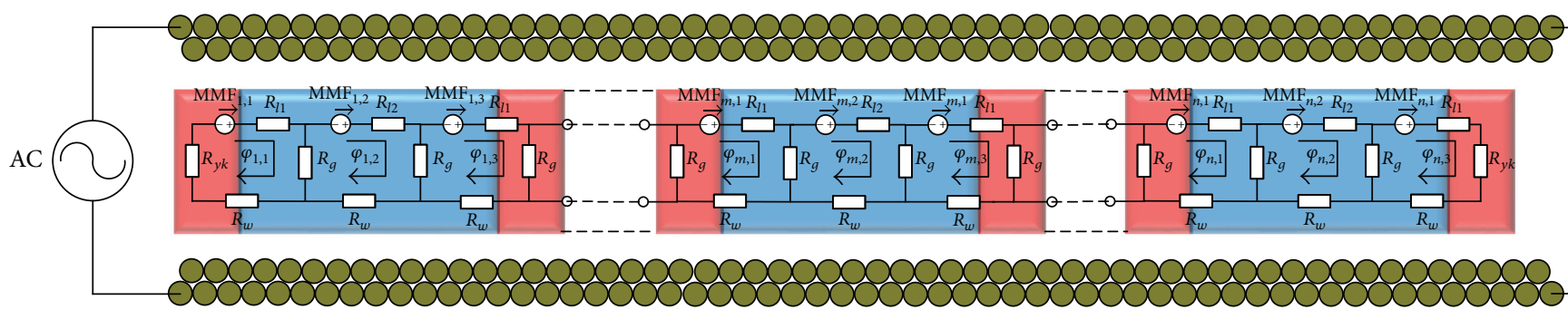

GMM
PM
Coil

FIGURE 7: Overall magnetic circuit model for the linear-arrayed GMA.

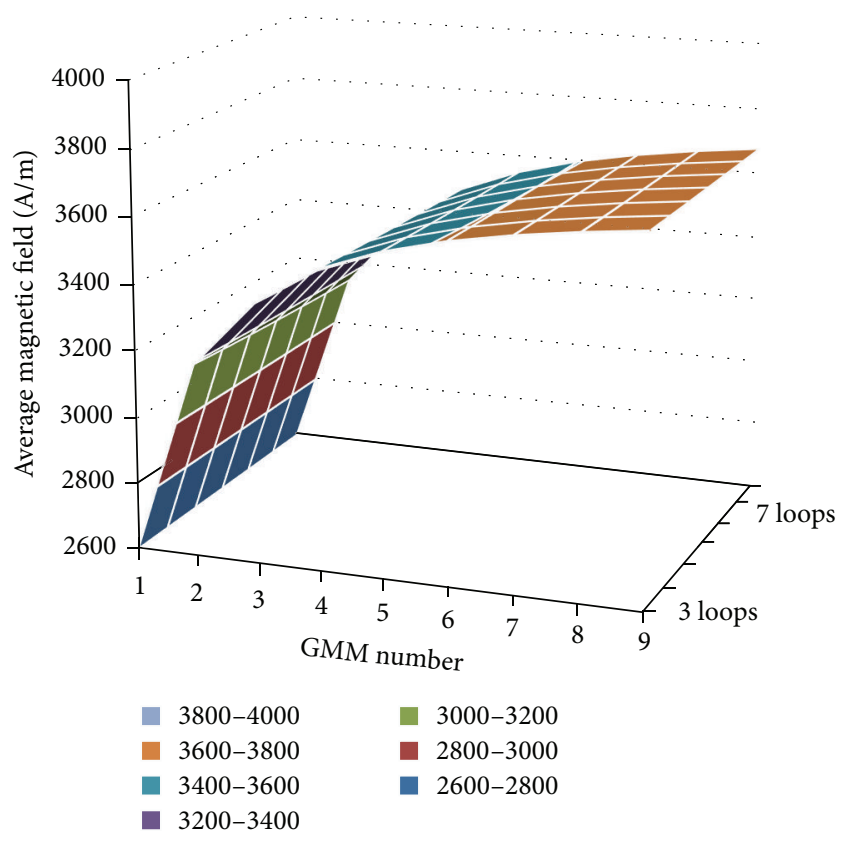

FIGURE 8: Average magnetic field strength variation when different GMM numbers and different loop numbers are employed.

According to Figure 8, the increase of GMM number helps to enhance the average field of GMM patches; in this way a considerable bias field is obtained using limited amount of PMs. The calculated field strength will increase slightly when there are more loops employed. This result has been proved also in single GMM case as the analysis of Figure 6.

3.1.2. Field Analysis. The setup for field analysis is similar to single GMM case, except for a geometrical iteration for GMM patches; a flow chart for this geometrical iteration method in a FEM platform is exhibited as Figure 9.

Based on the multi-GMM case loop model (10) and the extended field model of Section 2.2, the analysis results, when there is different number of GMM patches, are exhibited in Figure 10; in each GMM patch a 9-loop model is employed.

According to Figure 10, the field distribution described by loop model agrees with the FEM analysis in its total scale; however, instead of exhibiting a duplication property as loop model, the distribution curves obtained by FEM analysis indicate that the field distribution in each GMM patch will not duplicate the same pattern. This phenomenon arises since a certain PM will generate a bias field not only within its abutting GMM patches, but also in all the other GMMs along the rod. In another way, the magnetic field of certain GMM is determined not only by its abutting PMs but also by a field superposition from all the other PMs. This superposition makes the field distribution in different GMM patches vary slightly. In Section 3.2, an optimization will be conducted in order to relieve this unevenness.

A field analysis allows us to study more explicitly the unevenness of field distribution within GMM patches. The unevenness of axial magnetic field in GMM rod is evaluated via the following factor [11]:

$$
\eta=\frac{\sqrt{\left[(1 / N) \sum_{i=1}^{N}\left(H_{i}-H_{\mathrm{avg}}\right)^{2}\right]}}{H_{\mathrm{avg}}} \times 100 \% .
$$

Figure 11 exhibits a parametrical sweep result of GMM numbers, which is conducted to evaluate the average axial field distribution as well as its unevenness factor.

Figure 11 indicates a result in accordance with loop analysis; it also denotes that when there are more PMs included, the axial homogeneity will increase. The axial unevenness will drop to $2 \%$ when there are five GMM patches.

Supported by loop and field analysis, the proposed structure proves to adjust to the situation better when a larger bidirectional stroke is required: in that case, the total length of GMM bar has to be very long; a traditional structure with tube-like PM will make the overall structure very bulky; the structure with two PMs on the end of single GMM bar will make the axial field poorly distributed ascribed to its large aspect ratio.

In spite of the merits in this multi-GMM structure, there are some practical issues to be considered: when employing more PMs, the total length of this GMM-PM array has to be extended, which means more turns of coil and more energy consumption; too many patches of GMM will make it difficult to fabricate; the interfaces between alternate PMs and GMMs 


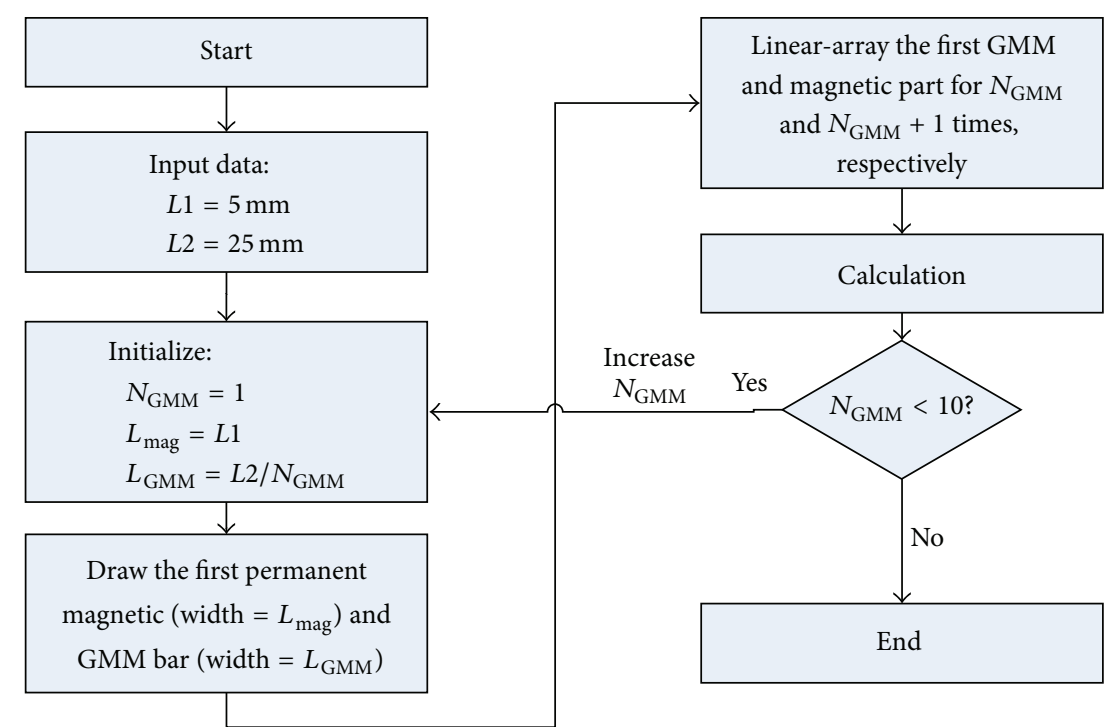

FIGURE 9: Flow chart for geometrical iteration of multi-GMM structure.

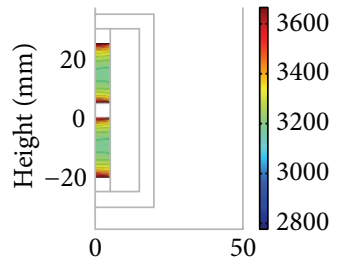

Radius position (mm)

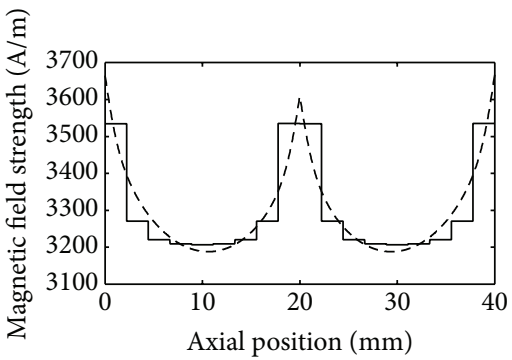

$$
\begin{aligned}
& \text { - Loop } \\
& --- \text { FEM } \\
& N=2
\end{aligned}
$$

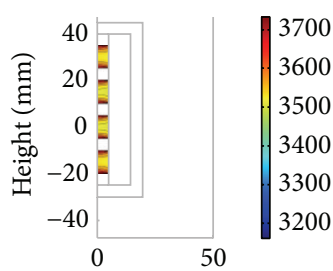

Radius position ( $\mathrm{mm})$

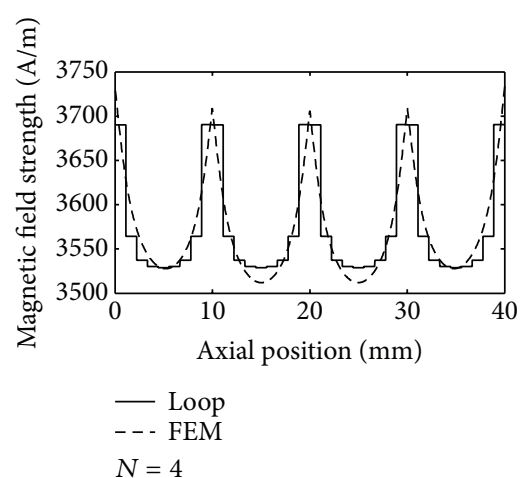

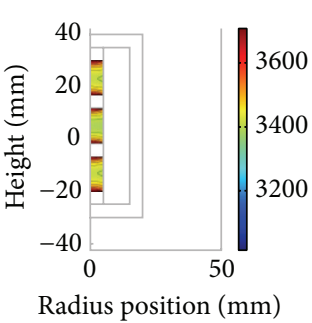

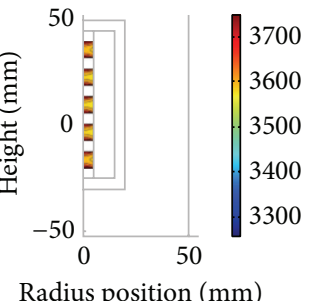

Radius position ( $\mathrm{mm})$

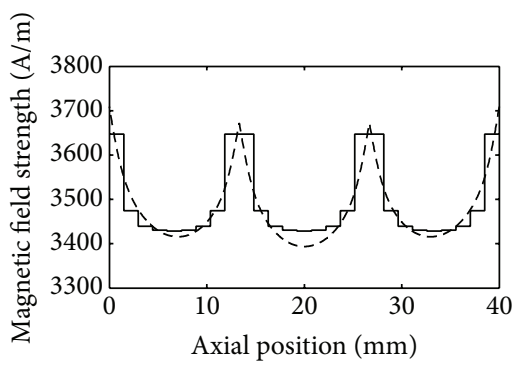

$$
\begin{aligned}
& \text { - Loop } \\
& -- \text { FEM } \\
& N=3
\end{aligned}
$$

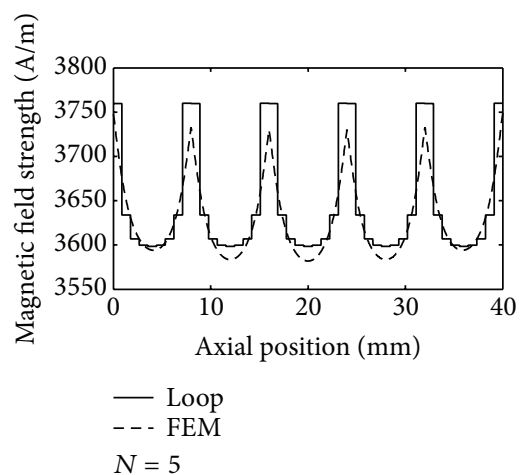

FIGURE 10: Magnetic field contour in GMM domain and axial distribution comparison with loop model.

are more vulnerable to crack after considerable duty circles and therefore degrade the mechanical strength as well as the reliability of overall structure. Therefore, an appropriate GMM number should be selected according to the required bias field, or fundamentally the expected stroke and power consumption. Figure 12 is a flow chart exhibiting the stages to determine the basic structure of SGMA from practical requirement.
3.2. Optimization. According to the field analysis result for multi-GMM case, the superposition effect deteriorates the axial field distribution in GMM; in this section, an optimization method is proposed to ameliorate these effects.

Indicated by field analysis results, there are three parameters that could be tailored in optimization. According to Table 1, among all the three factors, the changing of PM length concerns a little the field distribution in given total length; the 


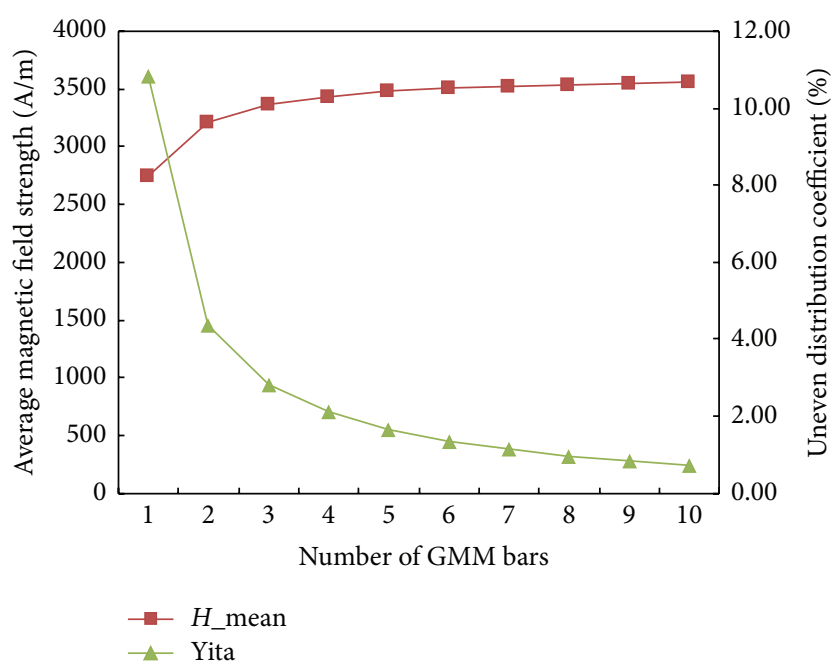

FIGURE 11: Average magnetic field and uneven distribution coefficient in different GMM bar number.

aspect ratio concerns only GMM length since its radii are already determined by output requirement. In this case, the optimization parameters are selected as the remanent flux density $B_{r}$ and length of each GMM patch $l_{\mathrm{GMM}, i}$. The resolution for this optimization is $0.01 \mathrm{~mm}$, which is also the resolution for common manufacture method.

The objective function for this optimization process is selected as the unevenness function detailed as (11). According to the stroke and power consumption requirement, the total lengths for GMM bar and PM are fixed; they denote two equality constraints:

$$
\begin{gathered}
\sum_{i=1}^{N} l_{\mathrm{GMM}, i}=L_{\mathrm{GMM}} \\
\sum_{i=1}^{N+1} l_{\mathrm{PM}, i}=L_{\mathrm{PM}} .
\end{gathered}
$$

In the above equation, $L_{\mathrm{GMM}}$ and $L_{\mathrm{PM}}$ denote, respectively, the total length of GMM and PM patches. In order to enhance the convergence velocity and seeking precision, the following constraints are also proposed to set a boundary in optimization process.

Based on observation of the curves in Figure 10, the axial field distribution of linear-arrayed GMA structure exhibits the following characteristics: to begin with, the axial distribution is symmetrical with its mid-perpendicular of the axis line; thanks to this evidence, the optimization of overall rod field distribution is simplified as the optimization for the upper or lower part of the rod; then the number of parameters to be optimized is halved by employing the first constraint:

$$
l_{\mathrm{GMM}, i}=l_{\mathrm{GMM}, N+1-i} \text {. }
$$

The alternative characteristics indicate that the average field intensity near the mid-perpendicular tends to be weaker. In this case, in order to obtain a uniform distribution, length of GMMs near the center is supposed to be shorter and therefore sets a lower boundary for parametrical optimization:

$$
\begin{array}{ll}
l_{\mathrm{GMM}, i}>l_{\mathrm{GMM}, i+1} & \left(i \leq N_{\text {half }}\right), \\
l_{\mathrm{GMM}, i}<l_{\mathrm{GMM}, i+1} & \left(i \geq N_{\text {half }}\right) .
\end{array}
$$

In the above equation, $N_{\text {half }}$ denotes the half number of GMM patches.

With the objective function detailed as (11) within the constraints of (13) and (14), the optimization of each PM length is conducted. The simulation results when GMM patch equals three to six are exhibited, respectively, in Figure 13, indicating that the proposed method helps to improve the axial field distribution in GMM patches. The red line in the figures denotes the minimum value of corresponding curves; this line is drawn to facilitate the uniformity comparison.

Table 2 lists a more detailed optimization result.

According to the above analysis, a general roadmap in optimal design of linear-arrayed GMA could be summarized as the following steps: to begin with, the number of GMM patches in given condition should be determined following the design procedure detailed in Figure 12; then, take the basic design as an initial condition, select an appropriate objective function, such as (11), together with the boundary conditions (12) to (14), and the optimized solution could be obtained by parametrical iteration.

\section{Experimental Validation}

Both magnetic field and displacement tests are conducted; the test system is exhibited in Figure 14.

The magnetic field intensity tests and displacement tests are conducted, respectively, using Tesla meter and eddy current displacement sensor. The test results are obtained by a data acquisition (DAQ) and restored in a master computer.

4.1. Magnetic Field Intensity Test. Based on the analysis and optimization results, a series of PM-GMM bar samples are manufactured, with different length for GMM and PM patches. The magnetic field intensity of SGMA is acquired by a Hall effect magnetometer and displayed on the Tesla meter; a specific frame is manufactured to monitor the close magnetic circuit. A sample combined three GMMs and four PMs are embedded within the frame. The samples of GMM-PM bars together with the steel frame are exhibited as Figure 15.

There is an orifice on the left side of the steel frame; in the practical test, the probe of Hall sensor will pass through the orifice and contact longitudinally with the surface of samples. According to the theory in computational magnetism, the field measured very close to the surface outside the material is equal to the intensity inside [32]. In each GMM patch in the sample five test points are evenly distributed along axial position; the field distribution is exhibited in Figure 16.

According to Figure 16, the field distribution of GMMPM bar sample is similar with simulation results in its changing pattern. The deviations between test and simulation field are ascribed to environment interference, material defects, and system error of measurements. 
TABLE 2: Optimization result of each PM length in different cases.

\begin{tabular}{lllllll}
\hline & $N=3$ & $N=4$ & $N=5$ & $N=6$ & $N=7$ & $N=8$ \\
\hline Initial unevenness factor & $3.03 \%$ & $2.24 \%$ & $1.77 \%$ & $1.45 \%$ & $1.21 \%$ & $1.15 \%$ \\
Optimized unevenness factor & $2.98 \%$ & $2.18 \%$ & $1.72 \%$ & $1.42 \%$ & $1.19 \%$ & $1.06 \%$ \\
\hline
\end{tabular}

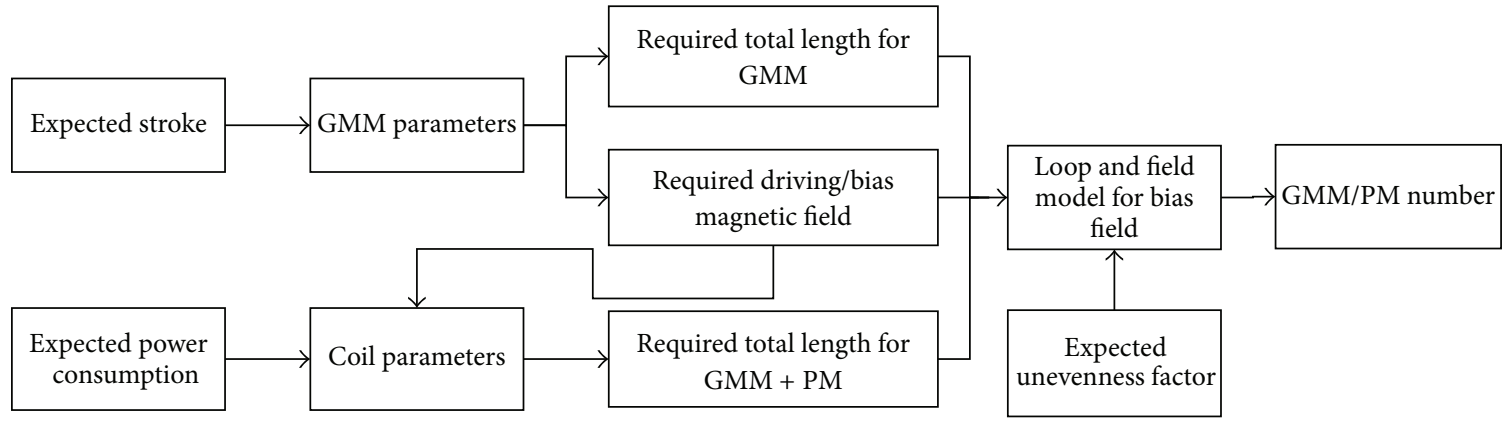

FIGURE 12: Design stages for the basic structure of SGMA.

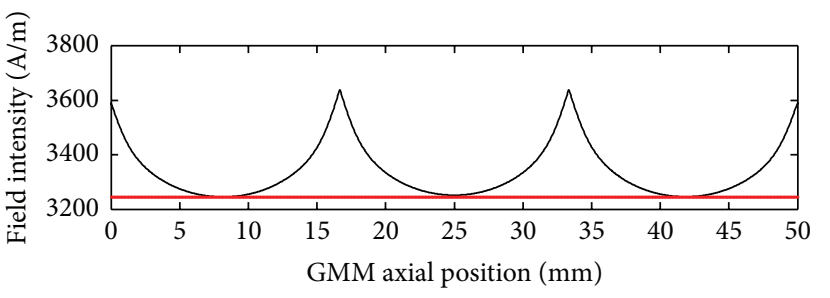

_ Optimization design

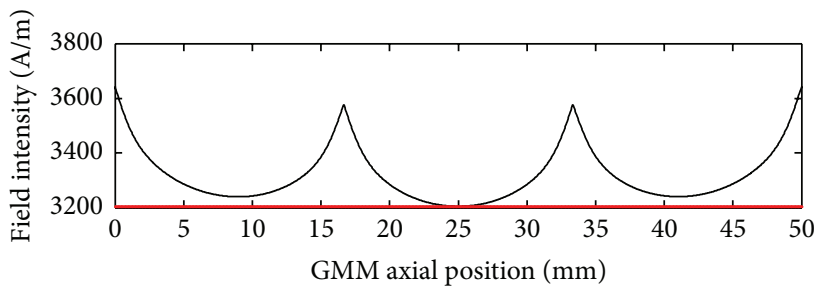

— Initial design

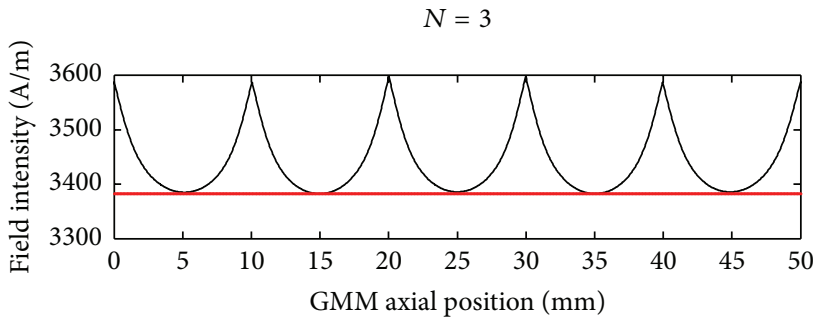

- Optimization design

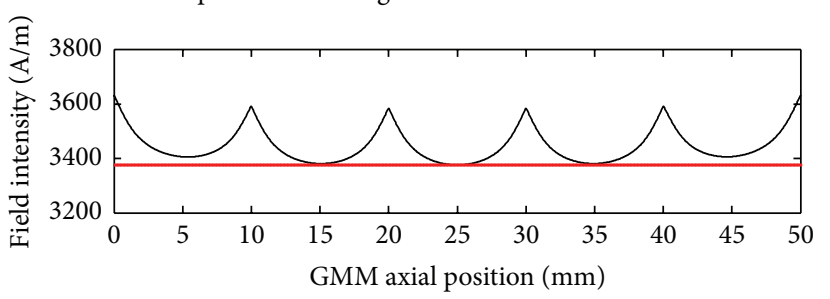

— Initial design

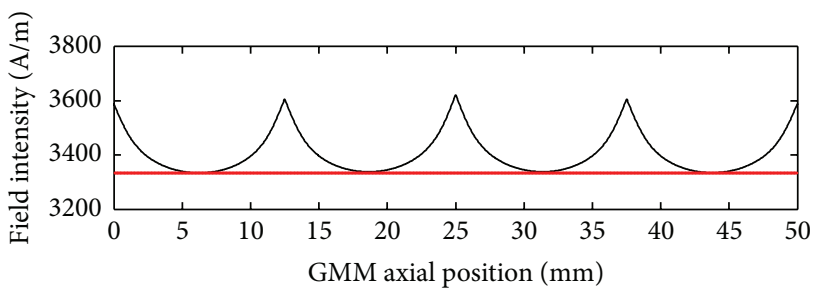

— Optimization design

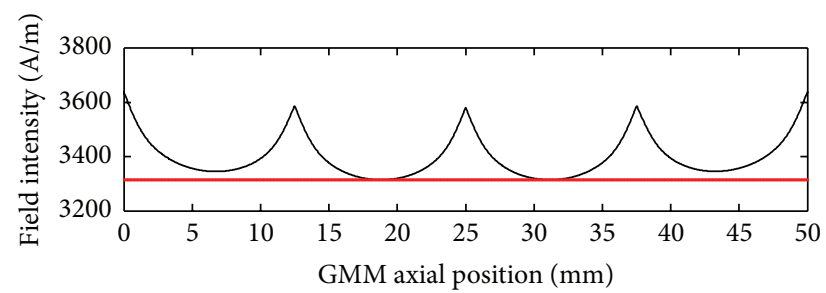

— Initial design

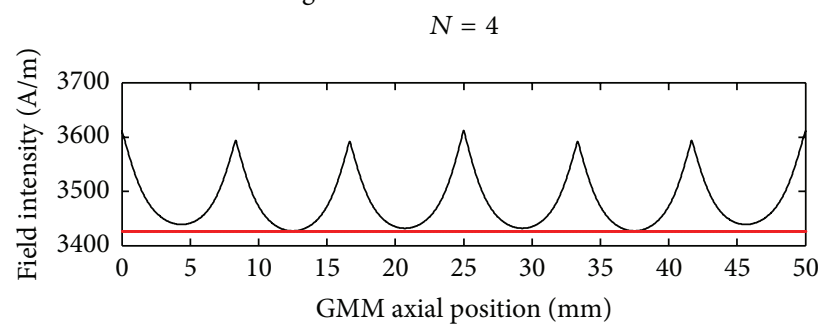

— Optimization design

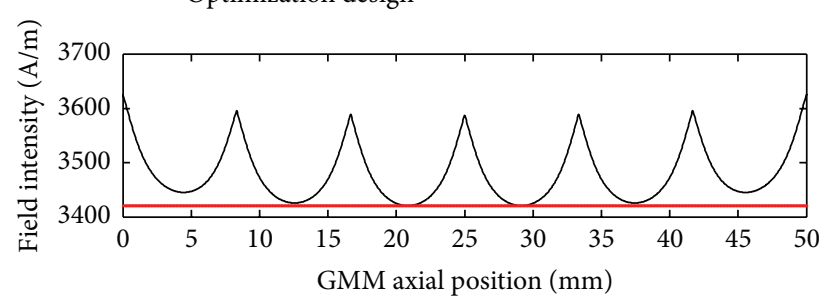

_ Initial design

$N=6$

$N=5$

FIGURE 13: Axial field distribution before and after optimization. 


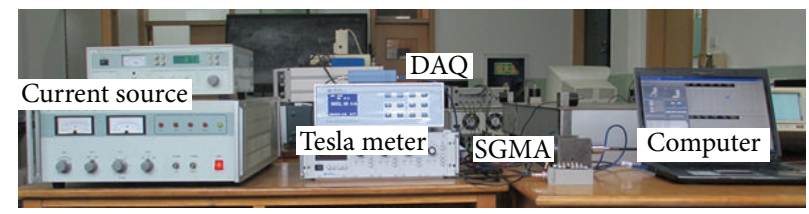

FIGURE 14: Setup for SGMA test system.

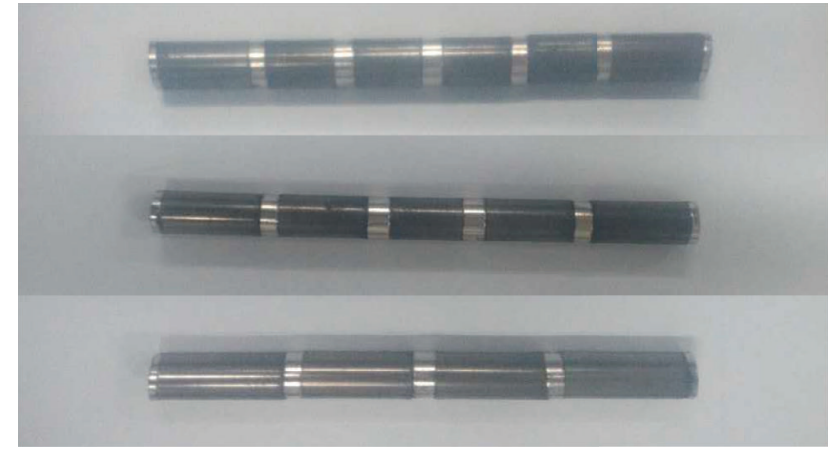

(a) Samples of GMM-PM bars with different GMM numbers

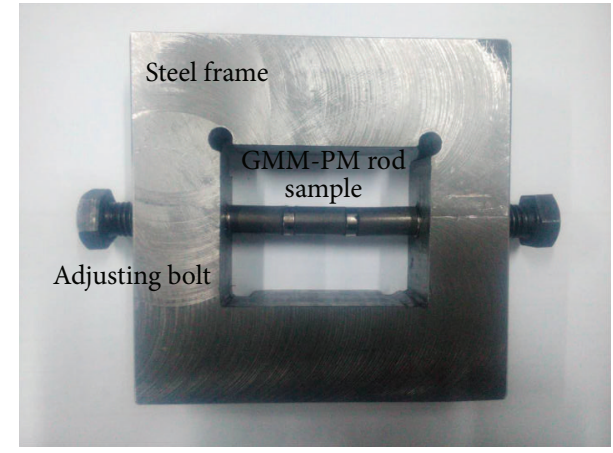

(b) Sample of a GMM-PM bar with steel frame

FIGURE 15: Samples of GMM-PM bars for magnetic field intensity tests.

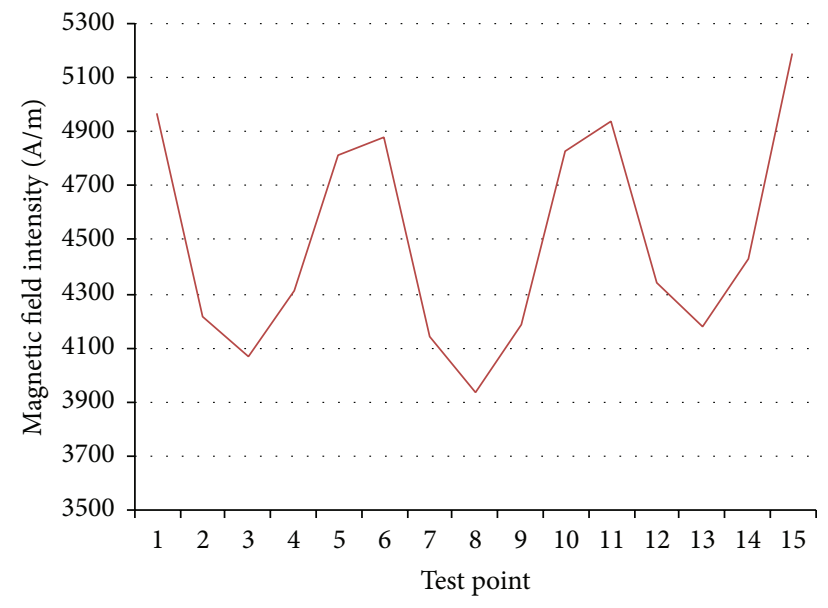

FIGURE 16: Magnetic field intensity test result.

4.2. Displacement Test. Mechanisms for GMM devices operation are fundamentally the process of interaction between multiple physics; its governing equation is therefore the coupling dynamics between electromagnetic field and solid mechanical field. The bridge between above fields is the constitutive relation between magnetization and magnetostriction $[28,33]$ :

$$
\lambda=\frac{3 \lambda_{s}}{2 M_{s}^{2}} M^{2} .
$$

In a static study, the magnetostriction calculated via (15) is regarded as the initial strain of GMM domain and therefore the overall elongation of GMM rod is derived by accumulation of magnetostriction within GMM domains.
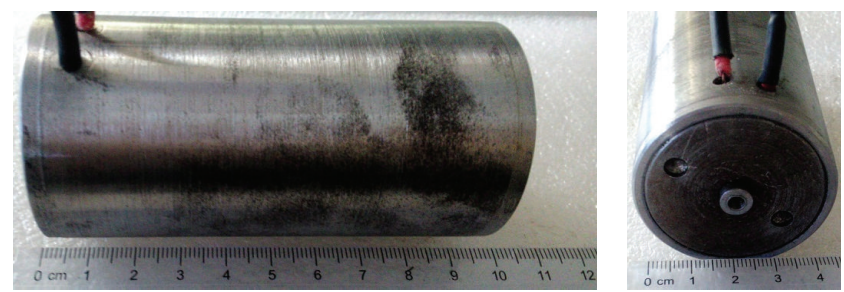

FIGURE 17: Photo of linear-arrayed GMA prototype.

Guided by design and optimization, a prototype is fabricated; there are three GMM patches included in the structure; after optimization, their lengths are $17 \mathrm{~mm}, 16 \mathrm{~mm}$, and $17 \mathrm{~mm}$, respectively. The photo of GMA is exhibited in Figure 17.

Displacement test is conducted by exerting a series of DC currents exerted directly on GMA; the test displacement curve and its comparison with simulated curve are detailed in Figure 18.

According to Figure 18, the saturated displacement of GMA is $33.9 \mu \mathrm{m}$ (positive) and $28.1 \mu \mathrm{m}$ (negative), which meets the requirement of our working condition; the specific linear-arrayed design is therefore valid; the simulated curve matches the test curve generally in quantity and agrees with its varying pattern; this result indicates the multicoupled model is amenable to analyze the output characteristics of proposed GMA and guide its design; the error between test and simulated curves is derived by multiple environmental factors: fiction between output shaft and house, thermal deformation of GMM rod, the leakage of magnetic circuit, and some unexpected factors which will be covered in our future works. 


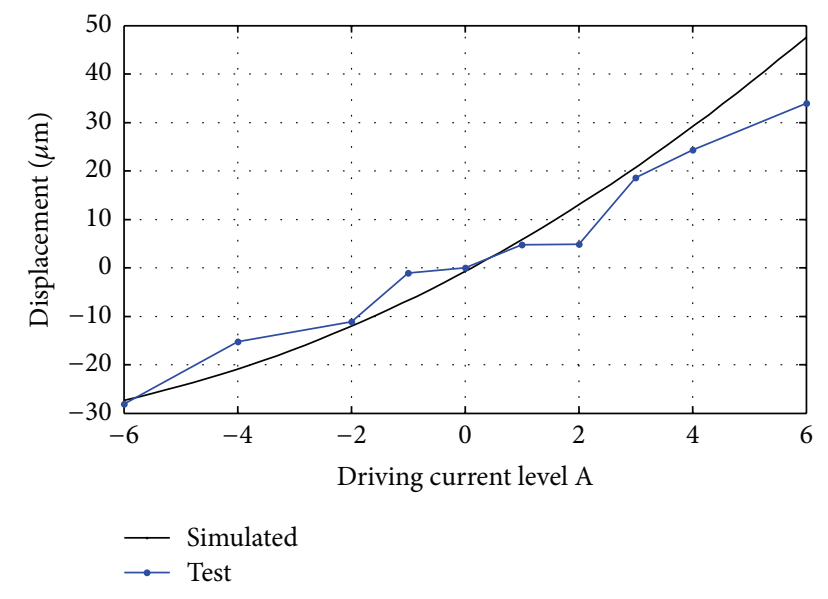

FIGURE 18: Test displacement curve and its comparison with simulated curve.

\section{Conclusion}

This paper covers the general design and optimization for the bias field of a stack GMA structure applied in novel EHSVs. The analysis methods and results in this paper could be applied in further design of SGMA structure. Some specific contributions and basic conclusion are listed as follows:

(1) Compared with traditional structure, a sufficient bias magnetic field could be exerted using less permanent magnet in SGMA; a multi-GMM case could improve the evenness of bias field distribution in SGMA.

(2) A concise loop model for the bias field of SGMA is established to cover the bias magnetic field distribution in GMM patches. Compared with traditional method, this model provides a concise and flexible way to calculate the uneven field in GMM.

(3) A specific superposition effect of bias field in SGMA is discussed; based on the proposed model, the design parameters are optimized to relieve this superposition effect.

(4) Samples with different GMM and PM numbers are fabricated and a prototype of proposed SGMA is fabricated; its tested magnetic field and displacement meet the practical requirement.

\section{Nomenclature}

MMF: Magnetomotive force provided by PM, A * turn

$R_{l}$ : $\quad$ Magnetic reluctance of the rod, $\mathrm{H}$

$R_{g}: \quad$ Magnetic reluctance of the air gap from GMM bar to wall, $\mathrm{H}$

$R_{w}: \quad$ Magnetic reluctance of wall, $\mathrm{H}$

$\varphi$ : $\quad$ Magnetic fluxes, wb

A: $\quad$ Magnetic vector potential, $w b / m$

$\phi: \quad$ Magnetic scalar potential, wb/m

$H_{i}$ : Magnetic field in each element, $\mathrm{A} / \mathrm{m}$

$H_{\text {avg }}$ : Average magnetic field, $\mathrm{A} / \mathrm{m}$

$L_{\mathrm{GMM}}$ : Total length of GMM patches, $\mathrm{m}$

$L_{\mathrm{PM}}$ : Total length of PM patches, $\mathrm{m}$ $\lambda: \quad$ Magnetostriction of GMM

$\lambda_{s}: \quad$ Saturated magnetostriction of GMM

$M_{s}: \quad$ Saturated magnetization of GMM, A/m

$M: \quad$ Magnetization of GMM, A/m

$A_{\mathrm{GMM}}$ : Section area of GMM rod, $\mathrm{m}^{2}$.

\section{Competing Interests}

The authors declare that they have no competing interests.

\section{Acknowledgments}

The authors acknowledge the financial support on behalf of National Science Foundation of China (no. 51275525).

\section{References}

[1] Z. Yang, Z. He, D. Li, G. Xue, and X. Cui, "Hydraulic amplifier design and its application to direct drive valve based on magnetostrictive actuator," Sensors and Actuators A: Physical, vol. 216, pp. 52-63, 2014.

[2] Z. Yang, Z. He, D. Li, J. Yu, X. Cui, and Z. Zhao, "Direct drive servo valve based on magnetostrictive actuator: multi-coupled modeling and its compound control strategy," Sensors and Actuators A: Physical, vol. 235, pp. 119-130, 2015.

[3] S. Karunanidhi and M. Singaperumal, "Design, analysis and simulation of magnetostrictive actuator and its application to high dynamic servo valve," Sensors and Actuators, A: Physical, vol. 157, no. 2, pp. 185-197, 2010.

[4] A. G. Olabi and A. Grunwald, "Design and application of magnetostrictive materials," Materials \& Design, vol. 29, no. 2, pp. 469-483, 2008.

[5] Y. Zhu, X. Yang, and X. Wang, "Development of a four-nozzle flapper servovalve driven by a giant magnetostrictive actuator," Journal of Systems and Control Engineering, vol. 229, no. 4, pp. 293-307, 2015.

[6] G. Xue, Z. He, D. Li, Z. Yang, and Z. Zhao, "Analysis of the giant magnetostrictive actuator with strong bias magnetic field," Journal of Magnetism and Magnetic Materials, vol. 394, Article ID 60356, pp. 416-421, 2015.

[7] C. C. Hong, "Application of a magnetostrictive actuator," Materials and Design, vol. 46, pp. 617-621, 2013.

[8] R. Joshi and R. Kadoli, "Magneto-mechanical coupled magnetostriction model for Terfenol-D actuator under step input," Procedia Materials Science, vol. 5, pp. 154-163, 2014.

[9] S. Chakrabarti and M. J. Dapino, "Hydraulically amplified Terfenol-D actuator for adaptive powertrain mounts," Journal of Vibration and Acoustics, vol. 133, no. 6, Article ID 061015, 9 pages, 2011.

[10] X. H. Liu, H. Zhang, X. L. Gao, and M. W. Chen, "Design and simulation analysis of giant magnetostrictive actuator," Materials Technology, vol. 30, no. 3, pp. 155-158, 2015.

[11] H. Zhang, T. Zhang, and C. Jiang, "Design of a uniform bias magnetic field for giant magnetostrictive actuators applying triple-ring magnets," Smart Materials and Structures, vol. 22, no. 11, Article ID 115009, 2013.

[12] M. J. Dapino, Nonlinear and hysteretic magnetomechanical model for magnetostrictive transducers [Ph.D. thesis], Iowa State University, Ames, Iowa, USA, 1999.

[13] G. Engdahl, Handbook of Giant Magnetostrictive Materials, Academic Press, San Diego, Calif, USA, 2000. 
[14] K.-Q. Qi, Y. Xiang, C. Fang, Y. Zhang, and C.-S. Yu, "Analysis of the displacement amplification ratio of bridge-type mechanism," Mechanism and Machine Theory, vol. 87, pp. 45-56, 2015.

[15] H. Steiner, M. Stifter, W. Hortschitz, and F. Keplinger, "Planar magnetostrictive micromechanical actuator," IEEE Transactions on Magnetics, vol. 51, no. 1, 2015.

[16] S. Wu, Z. Jiao, L. Yan, J. Yu, and C.-Y. Chen, "A fault-tolerant triple-redundant voice coil motor for direct drive valves: design, optimization, and experiment," Chinese Journal of Aeronautics, vol. 26, no. 4, pp. 1071-1079, 2013.

[17] F. Braghin, S. Cinquemani, and F. Resta, "A low frequency magnetostrictive inertial actuator for vibration control," Sensors and Actuators A: Physical, vol. 180, pp. 67-74, 2012.

[18] M. D. Noh and Y.-W. Park, "Topology selection and design optimization for magnetostrictive inertial actuators," Journal of Applied Physics, vol. 111, Article ID 07E715, 2012.

[19] L. Liyi, Z. Chengming, Y. Baiping, and L. Xiaopeng, "Research of a giant magnetostrictive valve with internal cooling structure," IEEE Transactions on Magnetics, vol. 47, no. 10, pp. 28972900, 2011.

[20] X. Mu, H. Tang, X. Gao, X. Bao, and J. Li, "Finite element modeling of magnetic bias field for magnetostrictive actuator," Chinese Journal of Magnetic Material Devices, vol. 45, pp. 6-10, 2014.

[21] Q. Xia, T. L. Zhang, J. A. Yu, and C. B. Jiang, “Topology option of bias magnetic field for magnetostrictive actuator," Materials Science Forum, vol. 787, pp. 295-299, 2014.

[22] J. S. Choi and J. Yoo, "Simultaneous structural topology optimization of electromagnetic sources and ferromagnetic materials," Computer Methods in Applied Mechanics and Engineering, vol. 198, no. 27-29, pp. 2111-2121, 2009.

[23] S. H. Cho, Y. Kim, and Y. Y. Kim, "The optimal design and experimental verification of the bias magnet configuration of a magnetostrictive sensor for bending wave measurement," Sensors and Actuators A: Physical, vol. 107, no. 3, pp. 225-232, 2003.

[24] X. Zhou, Z. Zhou, and Y. Ma, "Research on DC magnetic bias of power transformer," Procedia Engineering, vol. 29, pp. 452-455, 2012.

[25] R. C. Smith, "Inverse compensation for hysteresis in magnetostrictive transducers," Mathematical and Computer Modelling, vol. 33, no. 1-3, pp. 285-298, 1998.

[26] F. Braghin, S. Cinquemani, and F. Resta, "A model of magnetostrictive actuators for active vibration control," Sensors and Actuators, A: Physical, vol. 165, no. 2, pp. 342-350, 2011.

[27] J. Zheng, S. Cao, H. Wang, and W. Huang, "Hybrid genetic algorithms for parameter identification of a hysteresis model of magnetostrictive actuators," Neurocomputing, vol. 70, no. 4-6, pp. 749-761, 2007.

[28] Z. Yang, Z. He, D. Li, Z. Zhao, and G. Xue, "Dynamic modeling of a giant magnetostrictive actuator based on PSO," Journal of Applied Sciences, vol. 15, no. 2, pp. 311-315, 2015.

[29] A. G. Olabi and A. Grunwald, "Computation of magnetic field in an actuator," Simulation Modelling Practice and Theory, vol. 16, no. 10, pp. 1728-1736, 2008.

[30] K.-H. Hwang, S.-R. Kim, J.-H. Lee, D.-Y. Kim, Y.-H. Lee, and C.-M. Kim, "Design of a magnetostrictive actuator considering magnetic bias and ohmic heat," International Journal of Applied Electromagnetics and Mechanics, vol. 45, no. 1-4, pp. 867-871, 2014.

[31] T. Zhang, C. Jiang, H. Xu, and J. Mao, "Permanent-magnet longitudinal fields for magnetostrictive devices," Journal of Applied Physics, vol. 101, Article ID 034511, 2007.
[32] D. Jiles, Introduction to Magnetism and Magnetic Materials, Chapman \& Hall, London, UK, 2nd edition, 1998.

[33] Y. Liang and X. Zheng, "Experimental researches on magnetothermo-mechanical characterization of Terfenol-D," Acta Mechanica Solida Sinica, vol. 20, no. 4, pp. 283-288, 2007. 

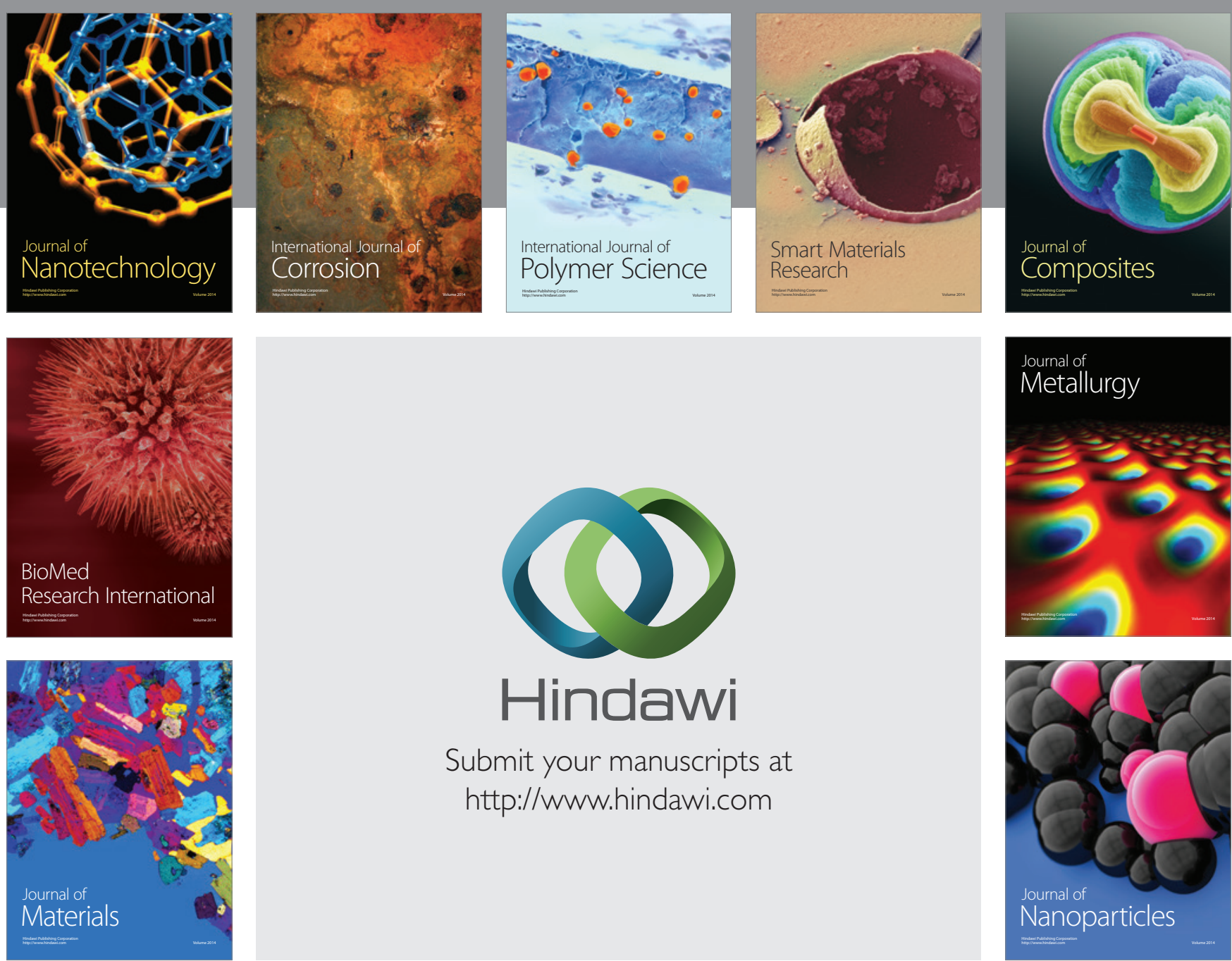

\section{Hindawi}

Submit your manuscripts at

http://www.hindawi.com

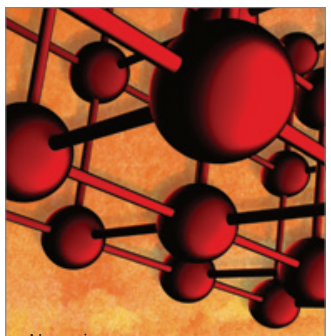

Materials Science and Engineering
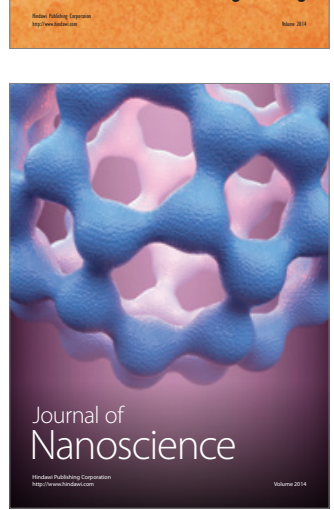
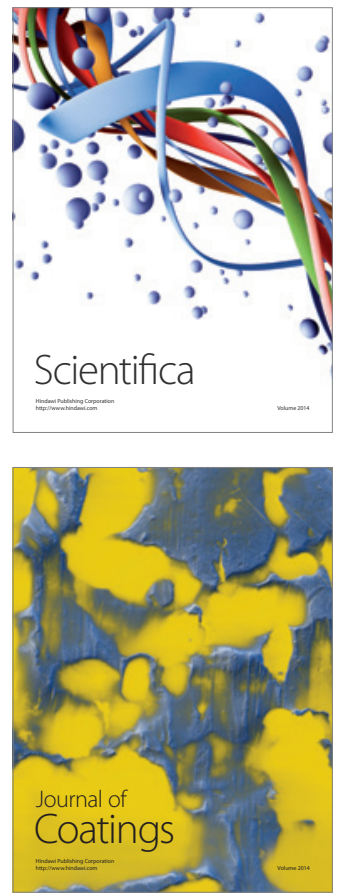
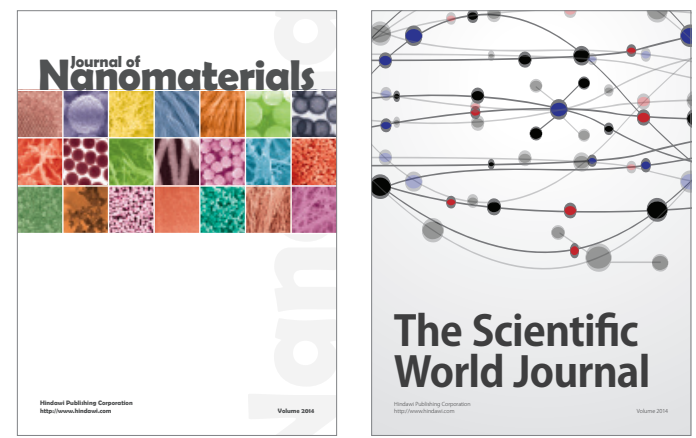

The Scientific World Journal
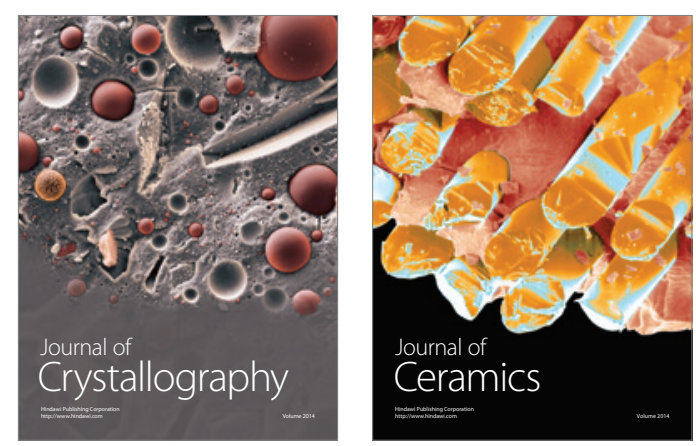
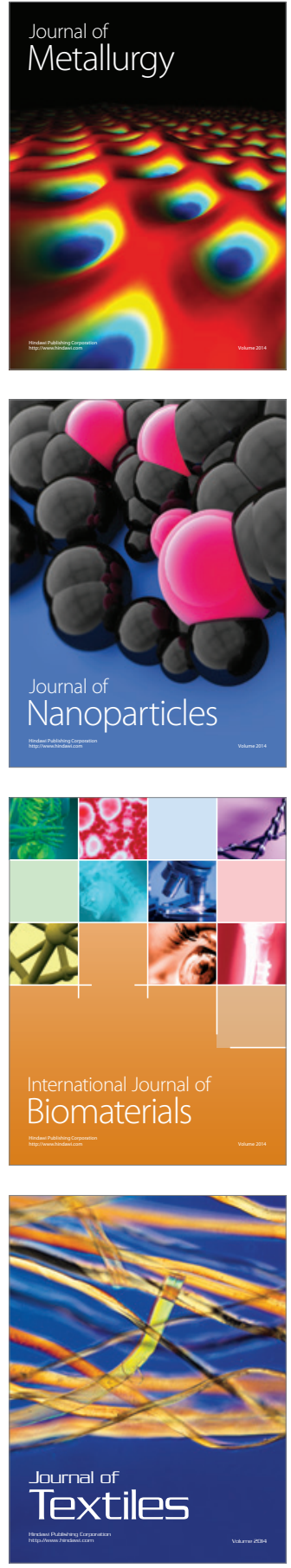